\title{
estado, regulación de los mercados y estrategia empresarial en américa latina: iberia, líneas aéreas de españa, en argentina y uruguay, 1966-1975
}

\author{
Javier Vidal Olivares \\ Universidad de Alicante (Espanha)
}

\section{RESUMEN}

El mercado internacional del transporte aéreo de pasajeros estuvo sometido a fuertes restricciones entre el final de la Segunda Guerra Mundial y el inicio de la liberalización, a partir de 1978. Las regulaciones instituidas por la IATA supusieron controles en las frecuencias de vuelo, en la capacidad de los itinerarios, altas tarifas y el establecimiento del principio de los convenios bilaterales. En estas condiciones la competencia entre aerolíneas era prácticamente imposible. Este trabajo muestra cómo Iberia, la empresa aérea española, puso en marcha mecanismos basados en el establecimiento de alianzas para eludir las regulaciones y obtener derechos de tráfico. Los ejemplos son los de Aerolíneas Argentinas y Pluna, la aerolínea de Uruguay. En ambos casos la estrategia de Iberia se basó en la cooperación técnica y financiera para aumentar sus ingresos por pasaje en los mercados del Atlántico sur. Esta fue una estrategia que Iberia también puso en marcha en otros mercados de América Latina durante este período anterior a la desregulación actual.

Palabras clave: aerolíneas, España, América Latina, mercados, regulación.

\begin{abstract}
The International market for air transport of passengers was subject to strong restrictions between the end of World War Two and the beginning of liberalization, started FROM 1978 in the United States. The regulations set up by the International Air Transport Association (IATA) included controls of flight frequencies and route capacities, high rates, and the establishment of the principie of bilateral agreements between governments. Under these conditions, competition among airlines was almost impossible.This arricie shows how the Spanish aviation company Iberia adopted mechanisms based on the establishment of alliances in order to circumvent regulations and to obtain traffic rights. The examples herein analysed are those of Aerolíneas Argentinas and of the Uruguayan firm Pluna. In both cases, Iberias strategy was based on technical and financial cooperation mechanisms in order to increase its revenues per ticket in the southern Atlantic regions.This strategy has also been put into practice by Iberia in other Latin America markets during that period previous to current deregulation.
\end{abstract}

Key words: airlines, Spain, Latin America, markets, regulations.

Este artículo forma parte de una investigación más amplia sobre la historia de la empresa de aviación comercial Iberia, Líneas Aéreas de España. El material de base 
El mercado mundial de transporte aéreo de pasajeros experimentó un crecimiento rápido e intenso entre el final de la II Guerra Mundial y la crisis industrial de 1973. Las condiciones en las que se desarrolló estuvieron marcadas por las reglas acordadas por la International Air Transport Association (IATA) y las aerolíneas designadas o de bandera. La asociación internacional funcionó como un cártel, en el que las compañías de bandera fueron las protagonistas de los transportes aéreos internacionales. Las reglas del bilateralismo se impusieron; los Gobiernos establecían las condiciones y negociaban las normas reguladoras del tráfico entre diferentes países. En la práctica, ello se tradujo en tarifas altas; control de capacidad en los itinerarios; establecimiento pactado de los puntos de entrada y salida de cada país. Pero, además, la mayoría del capital de las empresas de aviación debía ser, obligatoriamente, de propiedad de accionistas nacionales. Durante todo el período hubo elevadas barreras de entrada al negocio del transporte aéreo y las compañías de bandera se garantizaron el monopolio de las relaciones internacionales. ${ }^{2}$

La rigidez de las regulaciones no permitieron aumentar con facilidad la oferta e incrementar los tráficos, fuera de los propios mercados nacionales en los que operaban las compañías de bandera. Las estrategias basadas en la toma de participación y control de otras empresas de aviación fueron virtualmente imposibles y, en todo caso, se redujeron a participaciones minoritarias. Así los gobiernos establecían que las aerolíneas que participasen en los acuerdos bilaterales internacionales debían ser mayoritariamente propiedad de accionistas nacionales. En realidad esta restricción apenas tuvo importancia fuera de los Estados Unidos porque casi todas las compañías eran de propiedad estatal o estuvieron participadas por el Estado. Bloqueadas las posibilidades de adquisición de compañías de otros países, muchas empresas de aviación intentaron otras estrategias para conseguir derechos de tráfico al margen de los acuerdos bilaterales entre aerolíneas de diferentes nacionalidades.

procede de los Archivos Históricos del Instituto Nacional de Industria (ARGINI) y del Archivo General de Iberia, ambos en Madrid. Mi agradecimiento a los responsables de los Archivos (Elena Laruelo y Ana Sisniega para el primero y Ángel Jiménez Prados para el segundo). También quiero citar las ayudas recibidas de Elena Hernán del Centro de Documentación de Iberia por sus y facilidades para la obtención de información. Agradezco a los evaluadores de la Revista sus comentarios y sugerencias para mejorar el texto final.

Para el funcionamiento de los mercados aéreos entre 1945 y 1978 véase R. Doganis (1992), p. 24-42. 
Iberia fue una compañía europea que desarrolló esta estrategia en el área que consideraba, desde 1944, su mercado natural: América Latina.

Este artículo plantea, en primer término, las líneas básicas que siguió la compañía Iberia en la expansión de su red de itinerarios en América Latina. En segundo lugar, aborda la actuación específica de la compañía en el Cono Sur del continente; en particular, la acción de la aerolínea española con las empresas de bandera de Argentina-Aerolíneas Argentinas - y Uruguay - Pluna (Primeras Líneas Aéreas Uruguayas Nacionales). Por último, se llevan a cabo conclusiones generales, poniendo de relieve el fracaso parcial de la estrategia comercial de Iberia en el Río de la Plata a comienzos de la década de 1970. Dicho fracaso se explicaría por varias razones. La inestabilidad política de los gobiernos de la zona y los problemas de déficit público que arrastraban los presupuestos nacionales; las interferencias del Estado español en las medidas puramente comerciales de Iberia; y, finalmente, el escaso desarrollo de los mercados y el estancamiento de los tráficos en el cono sur de América Latina a comienzos de la década de 1970. Las propias reglas de funcionamiento del mercado aéreo internacional desde 1944 contribuyeron a hacer estériles los esfuerzos de la aerolínea española. El entorno institucional y, por tanto, el papel del Estado en las relaciones entre empresas de aviación comercial fue central desde el final de la Segunda Guerra Mundial y los inicios de la liberalización del mercado aéreo a partir de 1978 .

\section{La expansión de la red de vuelos de lberia en América Latina, 1946-1975}

A mediados de la década de 1950, la expansión del tráfico aéreo mundial que se había iniciado tras el final de la Segunda Guerra Mundial era un hecho. La principal área en expansión se centraba en el Atlántico Norte, en particular las rutas entre los Estados Unidos y Europa. Como señalaba un informe interno de la Compañía, a partir de los datos de IATA, en 1954 se realizaban 219 travesías atlánticas por semana en cada sentido. El flujo de pasajeros era ya de tal importancia que en el mismo año citado el número de viajeros trasatlánticos por aire era mayor que el de viajeros por barco. ${ }^{3}$ Comparativamente los mercados del Atlántico sur y medio atrajeron un menor tráfico al ser la naturaleza de los flujos

Archivo del Registro General del Instituto Nacional de Industria, ARGINI en adelante. Caja 325.4. 7-9/722. 
distinta. En el norte, la principal corriente de pasajeros se basó en turistas norteamericanos en tránsito a Europa; en el sur, en emigrantes.

A mediados de la década de 1950 Iberia reconocía que su oferta era insuficiente. La expansión de la demanda en los mercados que atendía era muy rápida. El lugar de transportador aéreo internacional, al que había optado tempranamente con las líneas americanas, necesitaba un nuevo impulso, con el objetivo de no perder tráficos a manos de otras compañías. Para ello era fundamental aumentar las disponibilidades de la flota para expandirse y crecer en América Latina. En la red del Atlántico Norte Iberia inició en 1954 la relación directa Madrid-Nueva York, con un éxito importante a lo largo del siguiente año, en que realizó tres viajes semanales. Pan American y TWA, las dos compañías norteamericanas de vuelos internacionales, llevaban haciendo el mismo itinerario con anterioridad, por lo que la competencia en los primeros años, y teniendo una diferencia notable de medios, fue un éxito relativo para Iberia. La compañía supo aprovechar la distribución de los turistas norteamericanos en Europa con su red continental.

En las rutas a América del Sur, Iberia dispuso de un trayecto con un alto nivel de ocupación. A Buenos Aires comenzó a volar en 1946; y desde 1947 a 1950 fue la compañía que más pasajeros transportó entre España y Buenos Aires. También desde entonces los enlaces de Santiago de Chile, Montevideo, Río de Janeiro y Sao Paulo fueron de los más rentables para la aerolínea española. ${ }^{6}$ Todo ello, como señalaba la compañía, cuando "nuestros aviones, a pesar de estar demodados y sin

"El aumento no es tan fuerte en el Atlántico medio ni en el Sur, ni sobre el Pacífico, cuyos tráficos están influidos por desplazamientos de masas de emigrantes para los que el avión no constituye un vehículo indispensable". Cf. Coyuntura y tendencias en el tráfico aéreo. Estudio sobre adquisición de material aeronáutico por Iberia. 12/11/1955. p. 5 .

Es importante señalar la cronología de la apertura de las líneas directas por el Atlántico Norte a Nueva York. Desde 1948: Air France, British Overseas Airways, KLM, Pan Am, Sabena, SAS,Trans Canada y Trans World. Desde 1949, Swissair. Desde 1951: EL AL y Linee Aeree Italiana.A partir de 1954 Iberia y, en el año siguiente, Lufthansa. Hay que vincular eso a la fuerte expansión del tráfico en esta área, sin duda la más importante del mundo en la demanda de pasajeros. Véase IATA, World Air Transport Statistics, 1956.

No cabe duda de que la política exterior franquista desempeñó un papel fundamental en la elección de Argentina como primer país americano en disponer de conexión aérea con España mediante la aerolínea de bandera. Los apoyos del presidente J. D. Perón a Franco eran un motivo decisivo para la elección. 
cabina estanca vienen llenos en las líneas de Suramérica". La reducción de1 $30 \%$ en las tarifas del tramo entre Buenos Aires y Madrid durante 1954-1956, debido a la utilización de los DC-4 ya superados técnicamente para los vuelos trasatlánticos influyó, decisivamente, en la rentabilidad de este itinerario para Iberia.

La decisión de establecer los recién adquiridos Lockheed Superconstellation en la ruta de NuevaYork - en detrimento de la Buenos Aires se hizo por motivos de prestigio y por el deseo de participar en la corriente de mayor tráfico de los mercados internacionales. Esto comprometió la situación comercial de la compañía en el resto de América Latina, ya que el número de pedidos acumulados para la fabricación de los Superconstellation dilató los plazos de entrega del nuevo avión." Al mismo tiempo, la atención al incremento de la demanda de viajeros en el mercado del sur del Atlántico se llevó a cabo con un material de peor calidad, lo cual dio ventajas a las aerolíneas competidoras, pese a que se trataba de itinerarios consolidados para la compañía española.

Iberia necesitaba disponer de cinco unidades de los nuevos Superconstellation para atender su plan de actuación en América y únicamente contaba con tres aparatos, que empezaron a operar a partir de septiembre de 1954. El objetivo era el mantenimiento de los tres vuelos semanales a NuevaYork desde Madrid; la disposición de un vuelo semanal a México, bien directo bien vía NuevaYork; un vuelo semanal a La Habana; otro semanal también a San Juan de Puerto Rico, Caracas y Bogotá y

Ibidem, p. 6.

En la estructura de tarifas de IATA, volar con modelos de avión retrasados tecnológicamente para la ruta en que operaban significaban reducciones de tarifas. Este era un incentivo pactado por todas las compañías para apoyar la renovación tecnológica de las flotas. Esta situación se volvería a plantear en 1961, cuando Iberia incorporó sus primeros jets - los DC-8 - pero seguía aún volando con Superconstellations a Río de Janeiro y Buenos Aires, con una tarifa denominada por IATA económica, que suponía un $36 \%$ menos que la general hasta Brasil;y el $25 \%$ a Uruguay y Argentina. Esta fue una propuesta de Iberia aprobada en la reunión de IATA celebrada en Paris el 19 y 20 de enero de 1961 a la que asistieron todas las compañías que intervenían en los tráficos del Atlántico sur. Las tarifas diferenciales entre reactores y aviones de hélice "dan una gran ventaja a Iberia en el momento actual en que volamos con Superconstellation, mientras que hay compañías que lo hacen con jet".Véase Actas del Consejo de Administración de Iberia, 26 de enero de 1961.

'Este modelo, puesto en servicio por la Pan Am en 1951 tenía autonomía suficiente para cruzar el Atlántico sin escalas, convirtiéndose en la aeronave de mayor envergadura y radio de acción mediante turbohélices para la navegación aérea interoceánica. 
también semanal a Río de Janeiro, Montevideo y Buenos Aires. Para rentabilizar las conexiones europeas, la línea de Nueva York podía extenderse a Roma o El Cairo; y la de Buenos Aires hasta Ginebra o Frankfurt. ${ }^{10}$ En este último caso, se buscaba la extensión de los itinerarios con el centro de Europa y el mediterráneo oriental que figuraban como objetivo de expansión de Iberia en su vinculación a las incipientes corrientes turísticas en la segunda mitad de la década de 1950.

La fuerte expansión del tráfico atendido por Iberia en la década de 1960 estuvo acompañada de un cambio de estrategia hacia América Latina. El rápido incremento de la demanda de tráfico en la década de 1960 1levó a Iberia a un crecimiento exponencial. La compañía explotó al máximo el auge del turismo procedente del norte y centro de Europa; al mismo tiempo que el tráfico interior se desarrolló a consecuencia de los efectos del Plan de Estabilización de 1959 sobre la economía española. El incremento de renta, tanto en el conjunto de Europa occidental como en España, benefició a todas las compañías de aviación comercial, especialmente Iberia. " La aerolínea de bandera española pasó a partir de 1966 de los dos millones de pasajeros transportados a alcanzar los seis en 1970. Ello representaba situarse a la altura de Alitalia y Air France, compañías de bandera de países que superaban en población y renta real por habitante a España.

El aumento del pasaje transportado por Iberia también se basó en los otros mercados en los que, desde la década de 1950, se concentró la acción de la compañía. En el Atlántico Norte se fue ganando volumen de pasaje, a pesar de la competencia de las grandes compañías norteamericanas y canadienses que, como TWA, Pan American y Canadian Pacific, volaban a Madrid y Barcelona. Este mercado representó para la española el $31 \%$ del total del pasaje americano en 1965; y respecto al volumen total de los viajeros desde los Estados Unidos a España ganó 12 puntos porcentuales, desde el $25 \%$ en 1961 al $37 \%$ en 1965. Estaba previsto poder alcanzar el $40 \%$ a fines de la década, manteniendo dos vuelos diarios entre Madrid y NuevaYork, y sin renunciar a negociar con los Estados Unidos nuevos itinerarios para Boston, Chicago, Los Ángeles o San Francisco. ${ }^{2}$

Coyuntura y tendencias.... p. 9.

Y también a su filial Aviaco, compañía que fue utilizada para asegurar el tráfico charter y las líneas internacionales que Iberia no consideraba de carácter troncal en su red.

Iberia. Gabinete de Planificación. Previsiones de demanda y necesidades de flota, 1965, p. 65 . 
En el área del Caribe, que representaba el $21 \%$ del tráfico de larga distancia, Iberia contaba con líneas a Puerto Rico, Caracas, Lima y Bogotá. Como la propia compañía aseguraba "Se trata de nuestro tráfico trasatlántico más saneado, tanto por su explotación, como por sus rendimientos y nuestra participación en el conjunto del mercado". ${ }^{13}$ Iberia había pasado a contar con el $53 \%$ de participación en 1961, en el tráfico de estas procedencias a España, al $62 \%$. Un porcentaje muy alto que se conseguía a pesar de la existencia de pool con KLM y VIASA, así como con AVIANCA, que disfrutaba de cupos de tráfico en cuarta y quinta libertad en San Juan de Puerto Rico y en Caracas. ${ }^{4}$ Indirectamente también concurría en este mercado la Pan American entre Puerto Rico y Lisboa. Cuba, por su parte, constituía un tráfico extraño en su comportamiento tras la Revolución encabezada por Fidel Castro. Para Iberia representaba, en 1965, el $4 \%$ de su tráfico transcontinental y, como sostenían los planificadores de su estrategia, "su evolución futura depende, en gran medida, de la forma en que se desarrollen los acontecimientos en el país a que sirve la línea. Se trata de un mercado que tiene una gran vitalidad potencial, en el que cualquier cambio de su estructura puede llegar a producir un espectacular desarrollo del tráfico". ${ }^{\text {s }}$ El fuerte auge del turismo norteamericano y europeo estaba detrás de este optimismo que el régimen castrista había ensombrecido, al impedir el crecimiento del flujo de viajeros en las proporciones adquiridas en otros destinos turísticos del mar Caribe.

Mención aparte merece el caso del tráfico con México. Para Iberia el mercado hispano-mexicano representaba el $13 \%$ del tráfico de largo recorrido. Si bien España carecía de relaciones diplomáticas con México, las reglamentaciones de IATA obligaban a un acuerdo bilateral en materia aeronáutica para regular las relaciones entre las compañías de aviación comercial de cada país. Todo ello no impedía la existencia de un acuerdo de pool entre Aeronaves de México e Iberia, que daba cobertura legal al

Ibidem, p. 67.

${ }^{14}$ Estas eran las compensaciones por la práctica de quinta libertad de Iberia en Bogotá. La quinta libertad suponía el derecho de una aerolínea nacional de llevar pasajeros de un segundo país a un tercer país, y constituía uno de los acuerdos básicos conseguidos en el convenio de Bermudas de 1946.Véase Stubbs, P. C. et alii (1980), p. 185-186; Brancker, J.W. S (1977); y Chuang, R.Y. (1972). Sobre la conferencia de Bermudas, D. Mackenzie (1991). Los tráficos de cuarta libertad suponían los derechos a recoger tráficos destinados por la aerolínea de un país de origen a otro segundo país, no necesariamente el propietario de la línea de bandera.

Ibidem, p. 69. 
tráfico entre ambos países y que únicamente necesitaba de la aprobación de las autoridades aeronáuticas. El tráfico entre México y España estuvo prácticamente en manos de Iberia que pasó del $56 \%$ en 1961, al $70 \%$ en 1965;y llego aseren 1962 del $100 \%$. $^{16}$ Todo ello con una competencia directa e indirecta de compañías de bandera poderosas, como eran los casos de Air France, Canadian, KLM y Sabena que presentaron el inconveniente de volar vía Nueva York o Montreal. Las cifras de previsión en el mercado mexicano se realizaban teniendo en cuenta "la cesión de oportunidades que hay que ir realizando, en beneficio de nuestro socio de pool Aeronaves de México" que se cifraban entre el 66 y el $70 \% .{ }^{17}$

En el caso de las rutas del Atlántico Sur, Iberia había concentrado el $14 \%$ de su tráfico de largo recorrido. Se trataba de un mercado con menor capacidad de expansión comparado con el del Caribe y Atlántico Norte pero ni por ello el grado de competencia era menor:

"En este mercado es seguramente donde se produce la rivalidad comercial más dura de todos aquellos en los que interviene nuestra compañía. Ello es debido a que en el mismo concurren un número muy elevado de compañías europeas y sudamericanas, algunas de las cuales, por no tener mercado propio, o gozar de menor prestigio entre el público de los diferentes países que atraviesan sus líneas, no dudan en acudir a prácticas desleales de competencia, enturbiando el mercado". ${ }^{18}$

Brasil era el país al que se acusaba de estar llevando a cabo una política de endurecimiento de las concesiones de tráfico, imponiendo cuotas y limitando las frecuencias. Varig, Aerolíneas Argentinas y, en régimen de quinta libertad Air France y British Airways, constituían las compañías que operaban las rutas de Brasil, Uruguay y Argentina, con las conexiones de Paragua y Bolivia ya que Chile se cubría por la ruta del Pacífico. En estos mercados del Cono Sur de América Latina, Iberia, a pesar de los obstáculos desarrollados por los gobiernos brasileños y de los problemas de las devaluaciones monetarias en los años sesenta, por parte de algunas economías de la zona, había ganado rápidamente flujo de pasajeros hacia sus líneas. En 1961,1a compañía sólo tenía el $23 \%$ del

Ibidem.p. 71 .

Ibidem.

Ibidem, p. 72 . 
total de pasajeros volados hacia España desde esta amplia subárea regional americana, mientras que en 1965 ascendía al 54\%.

En la década de 1960 hubo un crecimiento muy rápido para Iberia. Los directivos de la Compañía asumieron tempranamente que la introducción de cambios tecnológicos en las flotas comerciales y en cortos períodos de tiempo, conducía a la necesidad de desarrollar una nueva dimensión, de tamaño y estructura, de la empresa. La implantación de los modernos reactores DC-8 ya en 1959, cuando los Superconstellation de Lockheed apenas se habían amortizado en las flotas de las compañías aéreas, obligó a nuevas inversiones. La necesidad de ganar cuota de tráfico en el mercado mundial debía traducirse en fuertes inversiones en flota y mejoras en productividad, disminución de costes y obtención de economías de escala.

En el mercado nacional la posición de monopolio no suponía ninguna amenaza; pero para poder aumentar el pasaje transportado en Europa había que disponer de una flota nueva, como era el caso de los Caravelle, fabricados por la firma francesa Sudaviation. En el mercado norteamericano e intercontinental, sólo los DC-8 del fabricante Douglas, podían hacer frente al reto de la expansión para conseguir mayor cuota del mercado. ${ }^{19}$ Para la supervivencia de Iberia como empresa era básico mantenerse en esta área y enfrentarse a la renovación tecnológica que implicaba modificar su estructura interna.

"De no hacerlo así se corre riesgo no sólo de desaprovechar una coyuntura mundial altamente favorable, sino lo que es peor de perder - al menos en parte - los beneficios del esfuerzo hecho hasta ahora. Como ya se puso de manifiesto en 1960, la era de los aviones reactores precisa de un volumen mínimo de empresa, que Iberia está en camino de obtener, pero que aún no tiene". ${ }^{20}$

\footnotetext{
19 También la empresa Boeing con su modelo 707, pero para Iberia no era una opción. Desde mediados de la década de 1950 mantenía unas privilegiadas relaciones con Douglas y la aerolínea española buscaba la homogeneidad de la flota para mantener reducidos costes de mantenimiento. La alternativa británica, con sus jets De Havilland Comets, había resultado un fracaso para la propia BOAC en la década de 1950. Véase al respecto P. Lyth (1998), pp. 54-55.

Iberia. Gabinete de Planificación. Previsiones de demanda y necesidades de flota, p. 4. El grifo es nuestro. Iberia ya había realizado tres importantes reorganizaciones internas de su organigrama de gestión. La primera en 1955 tras el acceso a la presidencia de Tomás Delgado y después en 1962 y 1964, estas últimas vinculadas a ampliaciones de capital y a instancias del socio mayoritario de la compañía: el grupo industrial público INI. Sobre el INI véase F. Comín y P. Martín Aceña (1991).
} 
Si la aerolínea española no conseguía alcanzar las dimensiones adecuadas, otras compañías europeas tampoco parecían estar mejor ubicadas en los que se refiere a la escala que adoptaban los transportes aéreos mundiales. El tamaño de las empresas de aviación comercial norteamericanas, con flotas de gran capacidad y volumen de pasaje en constante crecimiento, obligaban a las europeas a defenderse mediante una política de alianzas. El objetivo fue uniformar sus flotas de aviones de gran capacidad para conseguir el mantenimiento común del material e incluso se llegó a contemplar el intercambio de aparatos y tripulaciones, a fin de disminuir costes operativos. En 1969 existían los grupos KUSS —integrado por KLM, SAS y Swissair - y ATLAS - Air France, Lufthansa, Alitalia y Sabena. Estas alianzas estratégicas tenían como objetivo el compartir el mantenimiento de flotas basadas en aeronaves de gran capacidad y reciente aparición en el mercado de la aviación comercial: los 747 de Boeing y los DC-10 del fabricante Douglas.

La compañía española no estaba integrada en ninguno de estos grupos, pero mantenía estrechas negociaciones con ATLAS. Las razones de su exclusión en el seno de la alianza no estaban claras. Oficialmente se argumentaba que "en el reparto de funciones entre las distintas empresas no obtuvo ninguna misión que desarrollar para el Grupo"." Para tratar de adoptar una posición fuerte en las negociaciones futuras de adhesión y enfrentarse a las condiciones del mercado, Iberia buscó fortalecerse como compañía y se centró en dos objetivos: la defensa a corto plazo de su posición en el mercado europeo y el despliegue de una estrategia de relanzamiento en Estados Unidos y en América Latina. Iberia buscaba ingresar en ATLAS a través de la acción americana "si conseguimos una posición en las Repúblicas americanas excepcional, (se podrá) poner a Iberia en condiciones de coordinarse con alguna compañía europea de cara al futuro. Es decir, esta política en Hispanoamérica era la plataforma de la política cooperativa en Europa, de la mayor trascendencia para Iberia". ${ }^{22}$ Añadamos también que la industria aeronáutica europea inició en estas mismas fechas un proceso de colaboración, que acabó con la rivalidad existente hasta entonces en las empresas de propiedad estatal. En 1969 se ponía en marcha el proyecto

ARGINI. Caja 325.4.7.9/4620. Política cooperativa en Hispanoamérica. Informe del INI al Ministro de Industria, 6 noviembre de 1970, p. 2

Carta de Jesús Romeo Gorría, Presidente del CA de Iberia a Claudio Boada, Presidente del INI, 14 enero de 1971. ARGINI. Caja 325.4.7.9/4822 (Informes) Exp. 4. 
Airbus cuyo primer aparato estaría en el mercado en $1972 .^{2}$

A comienzos de 1970 y con el apoyo expreso del Ministerio de Asuntos Exteriores español, Iberia desplegó su estrategia en el mercado americano. Se trataba de una política de cooperación con empresas de aviación que operaban en América Latina, otorgando ayuda técnica y financiera "para lograr su desarrollo y estabilidad económica y (a cambio) las empresas obtendrían de sus Gobiernos un trato preferencial para Iberia en materia de derechos de tráfico y ayudaría con sus redes comerciales en los distintos países a incrementar las ventas de Iberia". ${ }^{24}$ lista política de cooperación con compañías locales se complementó con otra basada en acuerdos con aerolíneas europeas - Alitalia y la portuguesa T AP - con el fin de reordenar, satisfactoriamente, para todas las empresas el tráfico del Atlántico sur hacia Europa. ${ }^{25}$ Como telón de fondo había que tener en cuenta la expansión de las compañías de aviación comercial de los Estados Unidos en el conjunto de América Latina, que amenazaba con desalojar al conjunto de las aerolíneas europeas en estos mercados. ${ }^{26}$

Iberia se reservó en esta estrategia la elección de su "política cooperativa" a partir de dos requisitos: la situación geopolítica y la inexistencia de compromisos con otras empresas europeas o norteamericanas. El plan era preciso y ambicioso, además de plagado de riesgos; pero se enmarcó en un contexto de cierta euforia por los resultados, muy saneados, de la explotación de la compañía. Además, el crecimiento de los tráficos en el mercado de la América del Sur dejaba entrever ciertas posibilidades de atracción de pasajeros para Iberia desde otras compañías, tales como Avianca,Varig y Aerolíneas Argentinas, como se desprende del Gráfico 1.

E. Chadeau (1996), pp. 369-376. Una visión crítica de los orígenes del consorcio europeo Airbus desde la perspectiva norteamericana en T.A. Heppenheimer (1995).

${ }^{24}$ Ibidem, p.3. Como ha sostenido Eduardo González Calleja, los años de 1969 a 1973 fueron la época dorada de la "política exterior económica" del régimen del general Franco, donde las actuaciones culturales o meramente diplomáticas quedaron relegadas a un segundo plano en beneficio del asesoramiento al desarrollo sin especiales connotaciones ideológicas. E. González (2000), p. 68.

25 Esta política de cooperación entre compañías europeas ya estaba siendo practicada entre Lufthansa y Lan-Chile; KLM con Viasa y Air France con Avianca. Para la historia de estas compañías véase P. Lyth y H. L. Dienel (1998)

${ }^{26}$ Además de Pan Am y TW A desde 1968 Braniff había incorporado la red de itinerarios de la compañía Panagra en América Latina, tras la adquisición de esta última aerolínea. 


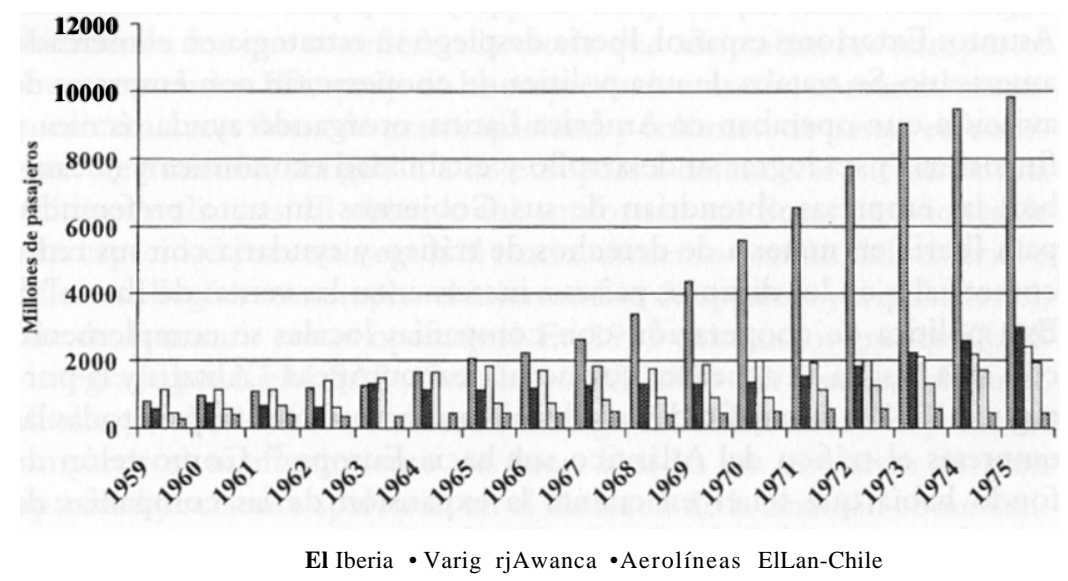

Fuente: IATA, Statistics. Elaboración propia.

\section{Derechos de tráfico versus cooperación técnica y financiera: Iberia en Argentina y Uruguay}

Con estos planteamientos, Iberia comenzó a poner en marcha su estrategia. En Argentina, la compañía española inició un plan de acercamiento a la aerolínea de bandera del país austral. Aerolíneas Argentinas se encontraba con un grave problema de disponibilidad de flota en la segunda mitad de la década de 1960. Tanto sus enlaces con Norteamérica, como con Europa, se hacían en inferioridad de condiciones con las compañías competidoras al disponer únicamente de anticuados aviones De Havilland Comet 1 , con elevados costes de mantenimiento. ${ }^{27}$ Aerolíneas Argentinas acumuló importantes pérdidas en su balance que tuvieron efectos desastrosos para el presupuesto asignado por el Estado argentino. La estrategia adoptada para eludir la inferioridad técnica no obtuvo los frutos esperados, como quedaba recogido en un informe interno de Iberia:

${ }^{27}$ El fracaso de la primera serie de los De Havilland Comet no permitió que las series posteriores tuvieran éxito comercial. Salvo BOAC, que compró la nueva serie Comet 4, el resto de las aerolíneas de bandera apenas hicieron pedidos de este avión.Véase P. Lyth (1998), p. 54. La hegemonía norteamericana fue decisiva en la fabricación de aviones de largo radio. 
"Los esfuerzos realizados por salir de esta situación, acudiendo a rebajas en las tarifas y otras prácticas ilegales de competencia, no hicieron más que agravar su desequilibrio financiero y comercial, obligándola a retirarse de la IATA, sin que por ello consiguiese una clientela sustancial, como para equilibrar sus balances anuales". ${ }^{28}$

Una posible salida a la situación pasaba por la modernización de su flota. La dificultad para obtener capitales obligaba al Estado argentino a recurrir a fuentes de financiación alternativas al presupuesto de la nación. Francia y Alemania, tradicionalmente con importantes intereses en la región, ofrecieron su ayuda a través de sus compañías de bandera, Air France y Lufthansa. Sin embargo, Aerolíneas encontró mayor sintonía con Iberia, no sólo porque le ofreció lo mismo que la compañía francesa y alemana sino sobre todo por "la comunidad de intereses existentes en la explotación de los mercados de América del Sur y Europa, unidos por las rutas del Atlántico sur". ${ }^{29}$

Para Iberia la ruta al Rio de la Plata y Santiago de Chile, a través de Brasil, era fundamental, sobre todo de cara al establecimiento de puntos estratégicos de apoyo básicos en el momento de entrar en explotación los aviones de gran capacidad tipo Boeing 747. Este planteamiento formó parte de un plan integral de la compañía para toda América:

"Operaciones de este tipo no pueden considerarse aisladamente sino que corresponden a un despliegue geopolítico de expansión de la Compañía que abarca los escenarios americanos en su conjunto. La magnitud de los intereses enjuego y las fuertes presiones realizadas por otras compañías interesadas en los mismos mercados, aconsejan actuar activamente para no verse desplazados por estas otras compañías que no regatearan ningún esfuerzo en sus intentos de penetración". ${ }^{30}$

Para conseguir una posición avanzada en el Cono Sur que permitiera la rotación de la flota de aviones de gran capacidad, Iberia debía lograr un acuerdo de cooperación con Aerolíneas Argentinas. Era necesario aumentar los derechos de tráfico en la ruta del Atlántico Sur. Para ello había que aumentar el número de interconexiones desde Buenos Aires, creando un hub en la capital del Río de la Plata. Estas acciones sentarían

Archivo de Iberia. Cooperación con Aerolíneas Argentinas, abril de 1967, Caja Aerolíneas Argentinas, 1968-1975, Estante 466.

Ibidem, p. 3.

39 $\quad$ Ibidem, p. 4. 
las bases para el futuro establecimiento de aviones de gran capacidad con perspectivas de rentabilidad.

Los acuerdos habrían de desarrollarse teniendo como contrapartida la financiación de dos aviones Boeing 707, cuyo coste era de 18 millones de dólares, incluyendo equipos opcionales, repuestos y accesorios. Los dos aparatos serían cedidos por la empresa española para su explotación a la compañía de bandera argentina, mediante un contrato de alquiler con opción a compra, integrándose en un acuerdo más amplio de cooperación entre ambas compañías. ${ }^{31}$ La operación de financiación de la compra de los aviones la llevó a cabo la empresa española para evitar problemas con IATA y con la legislación de aviación civil de Argentina que establecía que las compañías nacionales no podían ser financiadas por otras extranjeras. Fue, por tanto, la empresa española que en nombre propio compró los aviones para cederlos en arrendamiento a la argentina. Iberia se reembolsaba progresivamente del principal e intereses el coste total de la operación, que finalizaba el 1 de diciembre de 1975, por un monto general de 18.5 millones de dólares.

Para Iberia era fundamental conseguir llegar a un acuerdo más amplio que le permitiese concretar sus objetivos mediante los acuerdos suscritos por Aerolíneas. A mediados de 1968 propuso la firma de un Memorándum con compromisos más firmes. Ello era el paso previo para conseguir de la parte argentina un acuerdo sobre tráficos, rutas y estrategias comerciales conjuntas; y a la vez un claro intento de acordar una alianza entre compañías que comportase planificación común de servicios aéreos, coordinación de material, equipos y servicios e intercambio de flotas. Finalmente se planteó la creación de una comisión mixta para estudiar las medidas necesarias de colaboración en los terrenos acordados. ${ }^{32}$ La inestabilidad de los gobiernos argentinos y sus dificultades financieras retrasaron la aprobación del memorándum así como la puesta en marcha de las acciones que este encerraba. ${ }^{33}$ A principios

${ }^{31}$ Para Iberia la operación estaba muy clara:"debe recordarse que de no acudir Iberia a la financiación de estos dos aviones de Aerolíneas Argentinas sería automáticamente realizada su financiación por parte de Air France o Lufthansa, las cuales ya se han ofrecido en este sentido a Aerolíneas Argentinas, y continúan manteniendo en pié sus ofrecimientos". Ibidem, p. 6.

Archivo de Iberia, Proyecto de Memorándum Iberia-Aerolíneas Argentinas, julio de 1968 Caja Aerolíneas Argentinas, 1968-1975, Estante 466.

"Los sucesivos cambios en la administración de la compañía argentina, han ido retrasando que quedase plasmado por escrito en la totalidad el plan cooperativo, 
de 1969 la dirección de Aerolíneas señalaba que "el proyecto de memorándum compromete la acción de las autoridades aeronáuticas y por ello no correspondería que Aerolíneas Argentinas adquiriera ese compromiso sin conocer previamente la opinión de la autoridad aeronáutica argentina y contar con su conformidad". ${ }^{34}$

En octubre de 1969 volvieron a ponerse en marcha las conversaciones para establecer las bases de la cooperación entre ambas compañías sin alcanzar una alianza final. La española intentó relanzar el proceso para rentabilizar su esfuerzo de apoyo a la financiación de los dos Boeing 707, pero los dirigentes de la aerolínea argentina no dieron ningún paso adelante para formalizar los acuerdos. Las autoridades sólo autorizaron un incremento de las frecuencias de vuelo de Iberia a Buenos Aires pero en contrapartida pidieron insistentemente revisar las condiciones del pool con la española en las rutas europeas o de distribución de pasajes en vuelos de quinta libertad de Iberia y en itinerarios como el de Buenos Aires a Santiago de Chile. Como reconoció el Director Gerente de la española "en diversas ocasiones hemos cambiado impresiones a distintos niveles con los mandos de la Compañía argentina, pero el hecho cierto es que no cooperamos de manera que obtengamos los mejores resultados de nuestro esfuerzo de financiación". ${ }^{35}$

Los efectos del apoyo financiero a Aerolíneas Argentinas tuvieron un impacto directo en la cuenta de resultados de la compañía española. Los acuerdos de pool entre Iberia y Aerolíneas, establecidos en 1963 y vigentes hasta 1967 , presentaron un saldo favorable para la parte argentina de 1.630 mil dólares. Sin embargo, cuando se llevó a cabo el acuerdo de financiación de los dos Boeing 707, se modificaron los acuerdos del pool, en virtud del cual se incluyeron todos los tráficos de los vuelos de Iberia y Aerolíneas que tocasen en Madrid y Buenos Aires, incluidos tanto los de tercera y cuarta libertad, como los de quinta de extremo a

consecuencia lógica de la ayuda prestada en su día en la financiación de los dos aviones" afirmaba Lázaro Ros, Director Gerente de Iberia en carta a Director General de Navegación y del Transporte Aéreo en Madrid en 11 de octubre de 1969. Archivo de Iberia, Caja Aerolíneas Argentinas, 1968-1975, Estante 466.

${ }^{34}$ Archivo de Iberia, Carta del Brigadier Alberto Santamaría, Administrador General de Aerolíneas Argentinas a Lázaro Ros, Director Gerente de Iberia, Buenos Aires, 16 de enero de 1969. Caja Aerolíneas Argentinas, 1968-1975, Estante 466.

${ }_{35}$ Archivo de Iberia, Director Gerente. Nota para el Sr.Vicepresidente del Consejo. Política cooperativa con Aerolíneas Argentinas, 22 septiembre de 1970, Caja Aerolíneas Argentinas, 1968-1975, Estante 466. 
extremo de la ruta. El balance del pool cambió radicalmente a favor de la aerolínea española con los saldos siguientes: verano de 1967, 17 mil dólares; 1.080 mil dólares en el verano de 1969 y 126 mil dólares en el invierno de 1969/70. En total, la cifra de transferencias de Aerolíneas a Iberia por los saldos del pool entre abril de 1967 al 31 de marzo de 1971 , ascendió a 2.370 mil dólares. ${ }^{36}$

Dicho de otro modo, en 1967 Iberia pagó a Aerolíneas 409 mil dolares por el resultado de los acuerdos de ese año, mientras que, en 1969 , recibió de la compañía argentina US\$ 998.573. ${ }^{37}$ A partir de 1972 se modificó radicalmente la estructura del pool: se suprimieron los derechos de quinta libertad, y se acordaron limitaciones globales en los saldos del $3 \%$ del socio deudor. ${ }^{38}$ Aerolíneas llevaría a cabo seis vuelos semanales entre Buenos Aires y Madrid con los B-707 y la aerolínea española efectuaría la ruta con cuatro frecuencias semanales mediante los D C 8/63, aunque Iberia necesitaría de autorización de las autoridades aeronáuticas argentinas. La idea de Iberia fue la de mantener el pool. La compañía cedía algunas ventajas a Aerolíneas, como el aumento del porcentaje hasta el $3 \%$ del saldo deudor, pero sacaba fuera del pool los tráficos de quinta libertad, muy favorables a Aerolíneas Argentinas, ${ }^{39}$ e

${ }^{36}$ Archivo de Iberia, Conversaciones con Aerolíneas Argentinas. Informe del Director Comercial al Sr. Presidente del Consejo de Administración y Director Gerente sobre modificaciones en el pool comercial, 22 de febrero de 1972, Caja Aerolíneas Argentinas, 1968-1975, Estante 466.

${ }^{37}$ ARGini, Altos cargos, C. Boada, Caja 56, Exp. 1. 30/6/1970. Cooperación en Hispanoamérica. Rentabilidad directa para Iberia s/p.

${ }^{38}$ Aunque en abril de 1971 el porcentaje que se había barajado en las negociaciones, era del $5 \%$. En cualquier caso era un porcentaje superior al 1 o $1.5 \%$, lo normal en la época. Muchos acuerdos de pool tenían un límite en los ingresos que podían ser transferidos de una aerolínea a otra. El límite de la transferencia podía expresarse como porcentaje del total de los ingresos del pool o como porcentaje de las aportaciones individuales o de los ingresos recibidos. El objetivo de la limitación de las transferencias era asegurar a una aerolínea con un buen sistema de comercialización y ventas de pasajes no acabar transfiriendo grandes sumas de dinero a su ineficiente socio de pool. Como explica R. Doganis: "In the pool negotiations, the more succesful airline will try to have a very low transfer limit, while the weaker operator will want a high limit or no limit at all", R. Doganis (1992), p. 32-33 y R. Doganis (2001), p. 21-22. Los tráficos de quinta libertad, es decir los que se realizaban más allá de Madrid o de Buenos Aires por cualquiera de las dos compañías se habían repartido favorablemente para la aerolínea argentina en el año 1969-1970, representando un $89 \%$ respecto al $11 \%$ de Iberia. Añadamos que en el pool figuró el denominado Plus Ultra, vuelo que se mantuvo en un $50 \%$ de los ingresos y un $50 \%$ de los costes 
incrementaba su capacidad con el uso de aviones de mayor capacidad de pasajeros, como era el DC-8/63 frente al DC-8/52. ${ }^{40}$

Iberia mostraba un claro interés en estrechar progresivamente su alianza con Aerolíneas sin encontrar mucho eco en la compañía argentina. La operación de financiación de los B-707, gracias a la mejora de los resultados del pool, arrojó como resultado el aumento de los beneficios de Iberia en los tráficos del Atlántico Sur entre 1967 y 1972. Sin embargo, ello no supuso la creación de una alianza operativa que permitiese caminar hacia un tipo de colaboración más estrecha entre ambas compañías,

Desde el primer momento los gerentes de Iberia pensaron en una presencia mayor de Iberia en Aerolíneas, que los recelos del lado argentino y la propia situación política del país austral terminarían frenando."

El objetivo de rentabilizar la operación de arrendamiento de flota con aumentos de pasaje se cumplió; en cambio, el de conseguir un mayor grado de acuerdos por parte de Iberia para vincular la estrategia de Aerolíneas a la de la empresa aérea española, fracasó. Ya desde 1970, la empresa argentina inició contactos con Air France para explotar conjuntamente los nuevos Boeing 747 en las rutas del Atlántico sur desde Europa, aún a pesar de que el pool entre ambas compañías arrojó un fuerte déficit, que la propia compañía argentina se ocupó de denunciar. ${ }^{42}$

para cada aerolínea. Este vuelo fue explotado con avión de Aerolíneas en el trayecto Buenos Aires-Madrid-Zurich.

Q Que significaba pasar de 144 pasajeros a 210-250 por avión, aunque manteniendo los cuatro vuelos semanales.

No así por parte de los profesionales de la gestión de Aerolíneas. En agosto de 1969 el Gerente de Contabilidad y Finanzas, Vicecomodoro Juan Manuel Iglesias, había pedido a Iberia el organigrama de organización interna de la compañía española a fin de estudiarlo y compararlo con el de la argentina, Archivo de Iberia, Carta del Gerente de Contabilidad y Finanzas de Aerolíneas a Director Gerente de Iberia, Buenos Aires, 11 de agosto de 1969, Caja Aerolíneas Argentinas, 1968-1975, Estante 466. La inestabilidad política argentina entre el golpe de Estado militar contra el presidente Illia en mayo de 1966 y las elecciones constitucionales de mayo de 1973 que dieron la victoria a Héctor Cámpora, supuso un trasiego constante de funcionarios "con promedios ministeriales de apenas doce meses".Véase C. A. Floria; C.A. García Belsunce (1988), p. 196.

2 Archivo de Iberia, Oferta de Air France a Aerolíneas, Carta de Mario Aragoneses Moreno, representante de Iberia en Buenos Aires a Director gerente, 16 de diciembre de 1969, y Viaje del Gerente General de Aerolíneas, Comodoro Guillermo Ruzo, a París-Conversaciones con Air France, 5 de febrero de 1970, Caja Aerolíneas Argentinas, 1968-1975, Estante 466 . 
Al mismo tiempo, Aerolíneas planteó, en algún momento de fines de 1968, modificar su estrategia de penetración a Europa vía Lisboa, para aprovechar la fuerte corriente de tráfico existente entre Portugal y Brasil. Iberia trató de contrarrestar esta acción con la creación de un perita pool entre Alitalia, Aerolíneas, Iberia,Varig y Tap, que nunca llegó a cuajar. ${ }^{43}$ La crisis económica de 1973 y las inestabilidades políticas argentinas pusieron fin a un período de colaboración comercial que permitió ganar pasaje a la compañía española a cambio de financiación para la aerolínea argentina. En 5 dejulio de 1975 se produjo el traspaso de la propiedad de los dos aparatos a Aerolíneas por parte de Iberia, y se puso fin al contrato celebrado en 1967 por ambas empresas estatales.

El mercado del río de la Plata se complementaba con la acción concertada con Pluna (Primeras Líneas Uruguayas de Navegación Aérea), iniciada con la firma, en 31 de octubre de 1969, de un contrato de arrendamiento con opción a compra de un Boeing 737 por parte de la compañía uruguaya. A comienzos de 1970 Iberia y Pluna suscribieron un acuerdo de cooperación, aprobado por la presidencia de la República de Uruguay el 5 de marzo de 1970. El acuerdo implicaba asistencia técnica, estudio de la viabilidad de la empresa uruguaya y transformación de la compañía en un negocio rentable. Con anterioridad a la firma de los acuerdos, Iberia realizó, entre marzo y julio de 1969, un estudio exhaustivo de la compañía uruguaya. En aquella tarea la española detectó los puntos débiles y los enmarcó en un problema general:

"gran parte de las limitaciones actuales de Pluna, tienen como origen

\footnotetext{
${ }^{43}$ Sobre las maniobras de Aerolíneas para buscar un entendimiento con la TAP portuguesa,Véase Archivo de Iberia,Visita del Administrador General de Aerolíneas, Brigadier Santamaría a Madrid, Informe de Mario Aragoneses, representante de Iberia en Buenos Aires, 24 de octubre de 1968, Caja Aerolíneas Argentinas, 1968 1975, Estante 466. En lo que respecta al proyecto del denominado pentapool la compañía argentina nunca estuvo muy entusiasmada por el proyecto: "respecto al peligro que supone la actitud poco definida que en el seno de Aerolíneas existe sobre el proyecto de operación de las cinco empresas y la posición contraria de su Gerente Comercial, exteriorizada dentro de su organización, por lo que consideramos conveniente adoptar las medidas que juzguen oportunas a objeto de atenuar las gestiones iniciadas por Air France" lo que le hizo buscar apoyos en la compañía de bandera francesa. Este último texto es de Mario Aragoneses en Archivo de Iberia, Oferta de Air France a Aerolíneas. Ibidem. Para el pentapool y las conversaciones de Iberia con Alitalia, Archivo de Iberia, Relaciones con Argentina. Nota del Presidente del Consejo de Administración de Iberia a Director gerente, 30 de junio de 1969, Caja Aerolíneas Argentinas, 1968-1975, Estante 466.
} 
su naturaleza de Ente Autónomo, sometido a una serie de reglamentaciones de la Administración Pública que coartan, en cierta medida, su libertad de actuación y confieren un estilo burocrático a sus actividades, sin servir a nuestrojuicio, a un propósito definido. Estimamos fundamental que Pluna pueda ser dirigida con criterio empresarial, única forma de poder competir en igualdad de condiciones con otras compañías aéreas en el mercado internacional y alcanzar y mantener un nivel adecuado de rentabilidad, condición indispensable para asegurar la supervivencia del Ente". ${ }^{4}$

Los ejecutivos de Iberia plantearon un cambiojurídico de la compañía uruguaya con la creación de una sociedad anónima, controlada por el Estado.Esta nueva sociedad debía ocuparse únicamente de las actividades típicas de una empresa de aviación comercial internacional, además de separar los aspectos de infraestructura aeronáutica e incluir únicamente el handling ${ }^{45}$ como actividad comercial normal. La nueva compañía también debía dotarse de un organigrama sencillo y funcional; preparar un plan de acción y de inversiones; seleccionar nuevo personal; cambiar su sistema de contabilidad; implantar un adecuado sistema de control de gestióny, finalmente, mejorar el sistema de aprovisionamiento, el mantenimiento de flota y la gestión del personal. Por otra parte, Iberia se comprometía a establecer un asesor permanente en la dirección de la compañía uruguaya a fin de dirigir y coordinar el programa de asistencia. Se trataba, en definitiva, de crear una nueva compañía, organizada de forma completamente diferente a como se había desarrollado hasta entonces.

El contrato de tres años planteado por Iberia, incluía asesoramiento técnico y financiero a la nueva Pluna y perseguía su relanzamiento comercial mediante la dotación de un Boeing 737-2A, cuyo coste de compra correría a cargo de la compañía española que, a su vez, lo cedería en arrendamiento con opción de compra a la uruguaya. ${ }^{46}$ La cooperación buscaba evitar que otras compañías europeas captasen tráfico en Buenos Aires y Montevideo y permitía "para un futuro menos

\footnotetext{
"Archivo de Iberia, Informe sobre la Asesoría Técnica a Pluna, enero de 1972, Caja Pluna, 1971-1974, Estante 466. El subrayado es del original.

${ }^{45}$ Es decir sólo las operaciones en tierra de carga y descarga de equipajes, aprovisionamiento y limpieza de aeronaves.

${ }^{46}$ El coste del aparato se cifraba en 5'5 millones de dolares que pagaba la compañía Iberia mediante préstamo del Bank of América.
} 
próximo la posibilidad de utilizar los derechos de que Pluna pueda gozar en el hemisferio, como por ejemplo el ir a Nueva York". "Sin embargo los técnicos de Iberia descartaban que Pluna operase vuelos de largo radio, ${ }^{48}$ en parte porque no consideraban que fueran rentables, según sus estudios de previsiones de tráfico y, sobre todo, porque no era ese el objetivo fundamental asignado a Pluna en su esquema de organización comercial en el Atlántico Sur por los gerentes de la compañía española. La idea, por el contrario, era que la uruguaya operase como una empresa con una importante red regional, para distribuir los pasajeros procedentes de Europa y del resto de América y que fuera la propia aerolínea española la que operase la línea trocal con aviones de gran capacidad."

En efecto, la Dirección de Planificación de la compañía uruguaya había planteado en el verano de 1971 a la española la creación de nuevos compromisos, basados en el establecimiento de itinerarios, en los que sugería vuelos a NuevaYork y a Madrid y Roma, además de la compra de un B-707 que cubriera esas rutas. La respuesta de los ejecutivos de Iberia fue de prudencia en primer término pero, finalmente, desaconsejaron la realización de los proyectos. En primer lugar había que establecer claramente en un contrato las obligaciones del Pluna y el Gobierno uruguayo, en el que el segundo avalase a la primera en los

47 ARgini, Atos Cargos, C. Boada, Caja 56, Exp. 1. 30/6/1970. Cooperación en Hispanoamérica, p. 3.

* "Dada la situación financiera de Pluna, cuya compañía forma parte del Estado y la situación económica del país, no parece aconsejable iniciar una ruta a Europa o Estados Unidos, lo que supone unas inversiones importantes y pérdidas iniciales en los primeros años aun suponiendo que posteriormente fuese rentable". Nota de José María Gullón para el Director-Gerente, Madrid 23 de septiembre de 1971, Caja Pluna, 1971-1974, Estante 466.

* José María Gullón, Subdirector de Proyectos Especiales y el mejor conocedor dentro de la compañía Iberia de toda la operativa comercial puesta en marcha en América Latina por la empresa española, se manifestaba claramente respecto al papel que Pluna suponía en la estrategia de Iberia, por encima de los acuerdos políticos intergubernamentales: "Una compañía aislada, sin medios económicos ni créditos en el exterior, es probable que no pueda mantener su participación en el Atlántico. Consideramos, pues, que lo correcto sería estudiar el desarrollo de Pluna, no solamente con vistas a una línea a Estados Unidos y Europa, sino en su participación en el tráfico regional y la conveniencia de su expansión en ese tráfico, así como de su cooperación con otras compañías sudamericanas para el tráfico trasatlántico". En Archivo de Iberia, Nota de José María Gullón para el Director-Gerente, Madrid 23 de septiembre de 1971, Caja Pluna, 1971-1974, Estante 466. 
diferentes proyectos. Por otra parte, había que tener en cuenta la situación de los mercados del Atlántico Sur en su conjunto, así como la política comercial de las compañías aéreas competidoras. Finalmente la inestabilidad política del País respecto al futuro de los compromisos que eventualmente se adquiriesen, no aconsejaba acuerdos que significaran un volumen elevado de riesgos mayores de los ya adquiridos con el arrendamiento con opción a compra del B-737:

"La actual situación política del Uruguay coloca al país en unas condiciones de inestabilidad interna que no aconseja en este momento cualquier acuerdo comercial. Iberia debería esperar a las próximas elecciones que en el mes de noviembre se efectúan en el país para iniciar conversaciones que puedan significar un programa futuro de ayuda a Pluna". so

La tarea de consultoría técnica había previsto, en sus primeros pasos de propuestas de reorganización de la uruguaya, que el Gobierno de la República Oriental debía romper con su política de cielos abiertos y proteger a Pluna con los tradicionales derechos de bandera." Además, el Estado debía asumir una política activa de financiación de la compañía, no sólo cubriendo su déficit, como venía haciendo desde 1965, sino aportando capitales adicionales que pusieran a Pluna en situación de rentabilidad. ${ }^{52}$ Como tercer objetivo, era necesario modificar el estatutojurídico de la aerolínea, para hacerla menos dependiente de la administración y dotarla de flexibilidad para funcionar como una empresa de transporte competitiva. ${ }^{53}$ Finalmente, era necesaria la reorganización

Archivo de Iberia, Reuniones de la Comisión formada para estudiar el proyecto presentado por Pluna. Informe $\mathrm{n}^{\circ}$ 2. Consideraciones sobre la futura política de Iberia y Pluna, Madrid, 14 de septiembre de 1971, p. 1, Caja Pluna, 1971-1974, Estante 466

"Uruguay había mantenido tradicionalmente una política aeronáutica sin restricciones, en la que todas las compañías disfrutaban de derechos de quinta libertad y en la que apenas existían acuerdos bilaterales.

52 Además de otras medidas de complemento como eran el establecimiento de handling a terceras compañías, explotación de tiendas libres de impuestos y concesión del transporte de correo.

"3 Como sostenían los directivos de Iberia "Pluna está claramente diferenciada de lo que pudiera considerarse como un Organismo Estatal típico. Es una Empresa de Transporte Aéreo que compite dentro y fuera de las fronteras del país en un mercado internacional, con otras Compañías Aéreas que tienen una dinámica agresiva y 
de Pluna y su conversión en una empresa eficiente, con un nuevo organigrama, que incluyera mejoras retributivas del personal de vuelo y mantenimiento, ajuste de plantillas laborales y organización de un sistema de ventas y comercialización de pasajes.

El grado de cumplimiento de estos objetivos fue muy bajo. En el primero de ellos, el Estado uruguayo prácticamente no modificó su política de cíelos abiertos, mientras que trató de darle apoyo administrativo a la nueva Pluna diseñada por Iberia. El divorcio entre las dos políticas fue puesto de manifiesto por los técnicos de Iberia cuando evaluaron en enero de 1972 el fracaso de su acción en Pluna: "Lo que no puede hacerse es que haya dos políticas distintas y que se ignoren la una a la otra". ${ }^{44}$ Tampoco hubo apoyo financiero desde el presupuesto uruguayo para capitalizar la aerolínea nacional, ni Iberia consiguió modificar sustancialmente el esquema organizativo de la compañía de bandera uruguaya, a pesar de los medios desplegados y el interés de la compañía española. Las restricciones de la administración uruguaya en materia laboral y la propia inestabilidad política del País esterilizaron los esfuerzos por lograr cambiar la cultura corporativa de Pluna:

"El logro de unos objetivos se puede conseguir con una gestión acertada. Pero toda gestión requiere su tiempo. Los cambios continuos en la dirección de la Empresa imposibilitan el buen éxito de la gestión. Este ha sido el caso de Pluna donde en un período de un año han pasado tres personas distintas por la Dirección General del Ente" y "La plantilla de personal sigue siendo excesiva y, por otra parte, los sueldos existentes no permiten contratar al personal técnico especializado necesario".ss

Los problemas financieros fueron particularmente graves ya que el Gobierno uruguayo no estableció ningún plan a corto y medio plazo para capitalizar la compañía que, por otra parte, todavía siguió teniendo

que requieren, por tanto, una gran agilidad y flexibilidad en cuanto a las acciones a tomar por la Compañía, y que no deben estar subordinadas a una total estructura burocrática del estado" Archivo de Iberia, Informe sobre la Asesoría Técnica a Pluna, enero de 1972, p. 9, Caja Pluna, 1971-1974, Estante 466.

${ }^{54}$ Archivo de Iberia, Informe sobre la Asesoría Técnica a Pluna, enero de 1972, p. 29, Caja Pluna, 1971-1974, Estante 466.

${ }_{5 s}$ Archivo de Iberia, Informe sobre la Asesoría Técnica a Pluna, enero de 1972, p. 29-30, Caja Pluna, 1971-1974, Estante 466. 
la estructura administrativa de Ente: "En la actualidad la situación financiera de Pluna pone en grave riesgo cualquier acción que se quiera tomar sobre su continuidad y gestión". "Sólo podía hablarse de resultados positivos en los que se refiere a los ingresos de la compañía como consecuencia de la incorporación del B-737 a la flota de cuatro anticuados Vickers. Pluna pasó de ingresar US\$ 1.958 .572 en 1969 para US\$2.451.708 en 1970 , cuando desde el $1^{\circ}$ de mayo la nueva aeronave se tornó operativa. En 1971, los ingresos de Pluna ascendieron a US\$ 4.226.783, en una red que operaba con destinos a Buenos Aires, Rosario, Córdoba, Asunción, Montevideo, Punta del Este, Sao Paulo, Porto Alegre y Río de Janeiro.

A pesar de estos resultados, los problemas financieros de la compañía uruguaya acabaron paralizando la acción de Iberia, ya que ninguna de las medidas propuestas como urgentes por la empresa española se llevaron a cabo. En particular, el establecimiento de una plan de inversiones para cinco años y el saneamiento de la situación financiera y créditos del Gobierno a la empresa hasta que Pluna obtuviese rentabilidad en sus operaciones. Como señalaba la empresa española al final de su informe sobre la acción realizada en la aerolínea uruguaya desde 1969:

"Si estas medidas no son aceptadas por la Dirección General de Pluna y el Gobierno Uruguayo, y no son puestas en ejecución en el menor plazo posible, no consideramos viable la supervivencia de Pluna. Cualquier plan de soluciones parciales o limitadas que se intentare para un futuro desarrollo de Pluna, no podrá tener ninguna viabilidad a corto plazo". ${ }^{57}$

Desde abril de 1972, el B-737 no volaba. Aerolíneas Argentinas, empresa que revisaba los aviones de Pluna, retenía los motores del aparato por la acumulación de deudas de la uruguaya por valor de US\$ 400 mil. ${ }^{58}$ Al mismo tiempo, se había dejado de abonar con regularidad a Iberia los pagos por el arrendamiento del avión y sus repuestos, así como por la asistencia técnica. En julio de 1972, la deuda acumulada

${ }^{56}$ Ibidem, p. 30 .

${ }^{57}$ Ibidem, p. 35.

${ }^{58}$ Aerolíneas Argentinas ya había mostrado a Iberia su preocupación y recelo por la acción de la española en Pluna, ya que había supuesto una reducción del pasaje de la argentina a cargo de la uruguaya en las negociaciones sobre los acuerdos de pool en abril de 1971 . 
por gastos financieros y alquiler de la aeronave, asistencia técnica prestada e impuestos ascendía a US\$2.883.175. Hasta ese momento Pluna sólo había satisfecho con Letras del Tesoro los gastos financieros a Iberia hasta marzo de 1971, por un importe de US\$788.000. Ante las dificultades para hacer frente a sus obligaciones, la compañía uruguaya propuso liquidar la deuda pendiente mediante Bonos del Tesoro, a pagar a largo plazo, lo que finalmente rechazó la compañía española. ${ }^{59}$

Sin duda, la situación política y económica que vivía Uruguay desde mediados de 1968 favorecía la inestabilidad permanente del entorno institucional, haciendo muy difícil la continuidad de proyectos que dependían de decisiones gubernamentales. El presidenteJorge Pacheco Areco,en el poder desde fines de 1967, mantuvo alo largo de su mandato una política de estabilización, teniéndo que enfrentar a fuertes restricciones de crédito entre 1968 y 1970 por su política ambivalente frente a las propuestas de liberalización hechas desde el Fondo Monetario Internacional. Además de los problemas para afrontar la deuda externa, desde mediados de 1968 los conflictos con sindicatos y trabajadores acabaron generando fuertes tensiones sociales, agravadas por la intensificación de las acciones del movimiento guerrillero urbano conocido como Tupamaros. Las elecciones de noviembre de 1971, en medio de graves problemas económicos y políticos, implicaron un cambio de presidencia que pasó a manos de Juan María Bordaberry, cuya política económica no se manifestó claramente hasta febrero de $1973 .{ }^{60}$

En octubre de 1972, se reconocía al enviado de Iberia por el Ministro de Transportes del Uruguay, Uraburu Añon, que "para él, la situación a que se había llegado como consecuencia de la cooperación entre las dos compañías la responsabilidad era de la parte uruguaya, sin que Iberia tuviese culpa alguna". ${ }^{6}$ Los apuros económicos del Gobierno de la República Oriental elevaron el volumen de la deuda estatal y se reconoció que no existía una política definida para el futuro de la aviación comercial. ${ }^{62}$ Por razones políticas el Gobierno español presionaba a

${ }^{59}$ Archivo de Iberia, Deuda de Pluna al 31 de julio de 1972; y Política de cooperación y ayudafinanciera a Pluna, 9 agosto de 1972, Caja Pluna, 1971-1974, Estante 466.

${ }^{60}$ Un resumen de la política económica y social de la presidencia de J. Pacheco Areco y J. M. Bordaberry en H. Finch (1991), pp.213-220

${ }^{61}$ Argini, Caja 325.4.6.7/4991. Informe de la visita realizada a Pluna los días 3 al 5 de octubre de 1972, por J. M. Gullón. Subdirector de Proyectos Especiales de Iberia, p. 1.

${ }^{62}$ La presidencia de Juan María Bordaberry entre marzo de 1972 y 1976 se caracterizó por la toma del poder de facto por los militares y la crisis económica de fondo, que 
Iberia para mantener sus acuerdos con Pluna, a lo que los directivos de la empresa hispana contestaban con argumentos técnicos: la parte uruguaya no había cumplido con los puntos fundamentales del pacto, entre los cuales destacaba el de reestructurar la compañía como una sociedad anónima independiente dentro del conglomerado de la administración aeronáutica estatal, organizar una empresa moderna y apoyarla con créditos productivos; es decir, hasta obtener rentabilidad:

"Con las limitaciones actuales que se padecen en Pluna, sólo se podrán conseguir algunos pequeños objetivos de mejora de organización y funcionamiento de la empresa, pero nunca se podrá llegar a conseguir una empresa moderna, eficiente, que busca en el mercado del transporte aéreo un puesto destacado". ${ }^{63}$

En otros términos, las labores de consultoría y asesoría de los directivos españoles en Pluna no habían alcanzado el éxito porque no se habían podido llevar a cabo las medidas recomendadas y pactadas en el acuerdo. ${ }^{64}$ La deuda era a fines de 1973 de 3.300 .000 dólares que, finalmente, Iberia pudo cobrar parcialmente con la venta del B-737 a una compañía hondureña, tras la rescisión del contrato con Pluna en 29 de enero de $1974 .{ }^{65}$ El Gobierno uruguayo subrayaba "la buena voluntad de Iberia para lograr un acuerdo equitativo en términos menos onerosos para el país y de esta manera se da por finalizada una operación comercialmente iniciada en noviembre de 1969 y que no dio los frutos que sus promotores habían proyectado". ${ }^{66}$ El acuerdo cooperativo entre ambas compañías finalizaba en abril de 1974.

terminó acuñando el término bordaberrización como sinónimo de poder militar de facto frente a instituciones nominalmente civiles.

${ }_{63}$ Archivo de Iberia, Informe sobre la Asesoría Técnica a Pluna, enero de 1972, p. 28, Caja Pluna, 1971-1974, Estante 466.

${ }^{64}$ ARgini, Altos cargos. C. Boada, Caja 56, Exp. 7. Informe de la visita realizada a Montevideo para tratar de la devolución del avión B-737 y de la liquidación de la deuda de Pluna con Iberia, por José María Gullón, Subdirector de Proyectos Especiales y Jesús Muías, Director Económico Financiero, 20 Diciembre 1973, p. 3.

${ }^{65}$ J.Viniegra (1988), pp. 202-203. La deuda sería liquidada por el Gobierno uruguayo en un plazo máximo de tres años, mediante seis pagos semestrales consecutivos, equivalentes cada uno de ellos a una sexta parte del monto total de la deuda, con el incremento de un tipo de interés del $10 \%$ anual.

66 Diario La Mañana, Montevideo, 15 de febrero de 1974. 


\section{Conclusiones}

Iberia fue una compañía de aviación que, además de su mercado interior y el europeo, buscó tempranamente desarrollar tráficos de largo radio. América Latina concentró desde la década de 1950 los esfuerzos de la compañía para lograr convertirse en el mayor transportista aéreo entre Europa y el Atlántico medio y del sur. Además del establecimiento de su propia red en el subcontinente, la aerolínea de bandera española buscó tratar de consolidar su liderazgo a partir de la introducción de los aviones a reacción. Con la incorporación de una moderna flota en la década de 1960 y el fuerte crecimiento del tráfico de pasajeros por el auge del turismo, Iberia se planteó el incremento sustancial de su presencia en las compañías de aviación latinoamericanas.

El objetivo fue conseguir aumentar el volumen de negocio y asegurar rentabilidad a la compañía a fin de preparar la introducción en 19691970 de los nuevos aviones de cabina ancha, tipo Boeing 747 o DC-10. El Gobierno español, por su parte, buscó el impulso de la cooperación con América Latina por razones diplomáticas y políticas. En particular pretendió aumentar su grado de influencia en la región y disponer de una posición ventajosa frente a las negociaciones con la Comunidad Económica Europea y las iniciativas de Alemania y Francia para desarrollar una tecnología aeronáutica propia frente a la hegemonía de los Estados Unidos. El proyecto Airbus nacía entonces sin haber contado con España; el aumento del peso específico de Iberia en América Latina podía suponer la apertura de negociaciones en la participación en el proyecto como, finalmente, ocurrió.

La aerolínea española, de capital público, se centró en la búsqueda de alianzas de ámbito regional, que, a través de convenios de cooperación técnica, le aportasen tráficos a sus líneas troncales. En el caso de Aerolíneas Argentinas y Pluna, el mecanismo elegido se basó en la adquisición de flota para arrendar con opción a compra a las aerolíneas del Río de la Plata. A cambio debían de obtenerse mejores condiciones en los acuerdos de pool que implicasen el aumento de las frecuencias y la capacidad de los aviones entre Madrid y Buenos Aires y Madrid a Montevideo. De este modo, la aerolínea española se aseguraba la introducción de los nuevos aviones de cabina ancha que aumentaban, hasta en una tercera parte, la capacidad de la oferta existente, sin tener que acudir a otras concesiones en los acuerdos bilaterales que regulaban las condiciones del tráfico internacional, según las reglas vigentes de la IATA. Por otra parte, tanto la aerolínea argentina como la uruguaya disponían de nuevos 
aparatos que, eventualmente, iban a garantizar a Iberia una mejor redistribución de su pasaje intercontinental en los mercados regionales. Lógicamente la comercialización de pasajes en el tráfico de cabotaje interior por las compañías de bandera locales mejoraba la oferta de la propia aerolínea española. El fracaso parcial de la estrategia se debió a la inestabilidad política de la zona entre 1966 y 1974 que impidió la transferencia de recursos de la Administración uruguaya y argentina a sus compañías de bandera, así como el mantenimiento de una estrategia empresarial a medio y largo plazo.

En el caso de Aerolíneas las condiciones del llamado bilateralismo sólo permitieron una mejora de la posición de la española en el pool, así como un incremento de frecuencias y capacidad. En lo que hace referencia a Pluna, los directivos de Iberia pensaron que, dado que se trataba de un país con una política aeronáutica de cielos abiertos, la posibilidad de una alianza estratégica que implicara algún tipo de participación en la gestión podía ser importante de cara a tomar posiciones en el mercado aéreo regional. Sin embargo, se trataba de dos empresas de propiedad estatal donde era imposible, dadas las restricciones impuestas por las regulaciones aéreas nacionales e internacionales, el establecimiento de una toma de participación accionarial que pudiese conducir a la adquisición por parte de Iberia de la empresa uruguay. El Estado de la República Oriental no estaba tampoco en condiciones financieras de sanear la compañía, cambiar su estructura interna y prepararse para una eventual colaboración que fuese más allá del intercambio de derechos de tráfico y alianzas comerciales coyunturales con la aerolínea española.

Esta fue la consecuencia lógica del sistema de funcionamiento de los mercados aéreos internacionales entre 1944 y 1978 , en el que el juego de las relaciones internacionales y el papel del Estado en la regulación de la oferta, mediante la concesión de derechos exclusivos a las líneas de bandera, impidió la competencia entre las empresas y la estrategia de fusiones o adquisiciones de aerolíneas de diferentes nacionalidades. Estas fueron las consecuencias del funcionamiento de IATA y el carácter político de los acuerdos bilaterales, que se reforzaron, además, por la titularidad pública de la mayor parte de las compañías de bandera.

Los problemas encontrados con los gobiernos en el conjunto de América Latina en la década de 1960 y comienzos de los años 1970 fueron habituales en las líneas aéreas de bandera operadas por otros gobiernos de la zona. Iberia trató de mantenerse en la región limitando su acción a acuerdos de cooperación técnica o de consultoría. De esta 
manera salvaba la presión constante a que el gobierno español la sometía con el fin de utilizarla como instrumento de la política exterior, especialmente en el área de América Latina. Por otra parte, como empresa comercial de transporte necesitaba mantener una estrategia de permanente expansión en la región ya que estaba obligada a seguir bien ubicada en el Atlántico medio y meridional, uno de los pilares básicos de su actuación comercial.

El poder de negociación de Iberia con las compañías locales se basaba en la mayor envergadura financiera y en la mejor dotación de ejecutivos y técnicos, con una flota de tamaño medio, superior a la mayor parte de las compañías de aviación comercial de América Latina y de Europa. Pero los resultados de esta acción fueron, como se ha puesto de manifiesto, apenas parciales en los casos de Argentina y Uruguay entre 1966 y 1975 .

\section{Bibliografía}

Aparicio Gallego, J. (2000), "La regulación jurídica de la navegación aérea internacional", en VVAA, La Navegación Aérea en España. Madrid: Fundación AENA, pp. 53-95.

Brancker, J.W.S. (1977), IATA and What it Does. Leyden: A. W. Sijthoff.

Chadeau, E. (Ed.) (1995), Airbus, un Succès Industriel Européen. Paris: Institut d'Histoire de l'Industrie.

Chuang, R.Y. (1972), The International Air Transport Association. A Case Study of a QuasiGovernmental Organization. Leiden: A.W. Sijthoff.

Comín, F. y Martín Aceña, P. (1991), INI. Cincuenta Años de Industrialización. Madrid: Espasa-Calpe.

Diene1, H. L. (1998), "Lufthansa: Two german Airlines", en Lyth, P. J. y Dienel, H.L (Edited by), Flying the flag. European Commercial Air Transport since 1945. Londres: Macmillan, pp. 87-125.

Dierick, M. (1998), "KLM: An Airline Outgrowing its flag", en Lyth, P.J. y Dienel, H. L. (Edited by), Flyng the flag. European Commercial Air Transport since 1945. Londres: Macmillan, pp. 126-158.

Doganis, Rigas (1991), Flyng Off Course. The Economics of Internacional Airlines. Londres y Nueva York: Routledge. (Primera edición en 1985 en Harper Collins Academic). Se cita por la reimpresión de 2001.

Doganis, Rigas (2001), The Airline Business in the $21^{*}$ century. Londres y Nueva York: Routledge.

Durán, J. J. (1999), Multinacionales Españolas en Iberoamérica: Valor Estratégico. Madrid: Pirámide.

Finch, H. (1991), "Uruguay since 1930", en L. Bethell (ed.), The Cambridge History of Latin America. Vol.VIII, Latin America since 1930: Spanish South America. Cambridge: 
Cambridge University Press, pp. 195-233.

Floria, C.A;y C.A. García Belsunce (1988), Historia política de la Argentina contemporánea, 1880-1983. Madrid: Alianza Universidad.

González Calleja, E. (2000),"Cooperación en democracia: la ayuda al desarrollo de los gobiernos españoles hacia Latinoamérica, 1976-1992", Estudios Interdisciplinarios de América Latina y el Caribe.Vol. $11, \mathrm{n}^{\circ} 1$, pp. 65-88.

Hanlon, P. (1999), Global Airlines. Competition in a transnational industry. Oxford: Buttenworth-Heineman.

Heppenheimer, T. A. (1995), Turbulent Skies. The History of Commercial Aviation. Nueva York:John Wiley \& Sons.

IATA, (1979), How International Airline Passenger Fares and Cargo rates are Negotiated.An overview of the functions and organization of the Traffic Conferences of the International Air Transport Association, 7 páginas.

Iberia. Líneas Aéreas de España. Década 1960-1969. Madrid: Iberia, 1970.

Jarrett, Ph. (Ed.), (2000), Modern Air Transport. Worlwide Air Transport from 1945 to the Present. Londres.

Leary, W. M. (Ed.) (1995), From Airships to Airbus: the History of Civil ad Commercial Aviation. Washington: Smithsonian Institution Press.

Lyth, P. J, (1996), "Introduction: aspects of commercial air transport history", en P. J. Lyth (ed.), Air Transport.Aldershot: Scholar Press, pp. IX-XXI.

Lyth, P. J. (1997), "Experiencing turbulence: regulation and deregulation in the international air transport industry, 1930-1990", J. McConville (ed.), Transport Regulation Matters. Londres: Pinter, pp. 154-174

Lyth,P.J. y H. L. Dienel (ed.) (1998), Flying the Flag. European Commercial Air Transport since 1945. Londres: Macmillan.

Lyth, P.J. (1998),"Chosen Instruments: the Evolution of British Airways", en Lyth, P.J y Dienel, H. L. (ed.), Flyng the Flag. European Commercial Air Transport since 1945. Londres: Macmillan, pp.50-86.

Lyth, P. J. (1999), "Sky Wars: Conflicting Approaches to Air Transport Regulation in Europe and the United States, 1920 to 1990", en Lena Anderson-Skog y Olle Krantz (ed.),Institutions in the Transport and Communications Industries. State and Private Actors in the Making of Institutional Patterns, 1850-1990. Canton, Massachusets: Science History Publications, pp.94-111.

Lyth,P.J. (2002),"'Gimme a ticket on an aeroplane...'. The Jet Engine and the Revolution in Leisure Air Travel, 1960-1975", en Construction and Strengthening of a Tourism Industry in the XIXth-XXth century: technology, politics and economy. International prospectives. XIII International Economic History Congress, session 4, Buenos Aires.

Mackenzie,D. (1991),"The Bermuda Conference and Anglo-American aviation relations at the end of the Second World War",Journal of Transport History,Vol. XII, $\mathrm{n}^{\circ} 1$ (1991), pp. 61-73.

Mantegazza, A. (1998), "Alitalia and Commercial Aviation in Italy", Lyth, P.J. y Dienel, H. L. (ed.), Flying the Flag. European Commercial Air Transport since 1945. Londres: Macmillan, pp.159-194. 
Morrison,S.A.Winston,C. (1995), The Evolution of the Airline Industry.Washington: The Brookings Institution.

Neiertz, N. (1998), "Air France: an Elephant in an Evening Suit?"P. Lyth y H.L Dienel, Ed. Flying the flag. European Commercial Air Transport since 1945. Londres: Macmillan, pp. $18-49$.

Pattilo,Donald M. (1998), Pushing the Envelope.The AmericanAircraft Industry. Ann Arbor: University of Michigan Press.

Radnoti, G. (2002), Profit Strategies for Air Transportation. Nueva York: McGraw -Hill.

Sochor, E. (1991), The Politics of International Aviation. Londres: Macmillan.

Stratford,A. H. (1973), Air Transport Economics in the Supersonic Era. Londres: Macmillan St. Martin's Press (Second Edition).

Stubbs, P. C;Tyson, W. J. y M. Q. Dalvi (1980), "The Economics of Airlines", Transport Economics. Londres: Alien \& Unwin, pp. 185-204.

Viniegra Velasco, J. B. (1988), Iberia. Cronología de seis décadas. Junio 1927-Junio 1987. Madrid: Iberia, 1988. 